Prof. F. E. Simon discussed the process of heat conduction in solids, emphasizing the role of the mean free path of the heat waves. Reduction of the mean free path by disturbing the lattice, either physically (mechanical distortion, use of supercooled disordered phases) or chemically (alloying), gave a very low thermal conductivity at low temperatures.

In the subsequent general discussion, stress was laid on the possibilities of an insulator composed of powder or fibres with gas at low pressures in the interspaces.

EZER GRIFFITHS

\section{SOME NEW ANISOTROPIC TIME EFFECTS IN RHEOLOGY}

\section{By S. M. FREEMAN and K. WEISSENBERG British Cotton Industry Research Association, Didsbury, Manchester}

\begin{abstract}
$\mathrm{N}$ rheology we study the development in space and Iime of the mechanical variables, that is, of the stresses and strains which co-exist in a material in every differential cell, and at every instant. Our concern here will be the comparison between different directions in space, and we shall speak with respect to any quantity, or development, of isotropic symmetry to denote identity in the various directions, and of anisotropic symmetry to denote differences from one direction to another.
\end{abstract}

Let a material be observed as it passes under some mechanical action through a series of rheological states, and let all such states be divided into sheared and shear-free states according as we can, or can not, find a tangential component of the mechanical variable under consideration on a plane of at least one orientation. It can then be shown that any passage between a shear-free and a sheared state will necessarily exhibit some anisotropic development in time, even if the material itself is of the isotropic kind, that is, shows the same response to mechanical actions in all directions of space when tested in a ground-state, where it is at rest and free from externally applied forces. (It follows from symmetry considerations that every shear-free state must be isotropic, and every sheared state anisotropic; and hence, any passage between the states of the two classes must exhibit anisotropic time effects with respect to the mechanical variable which possesses a tangential component.) We shall restrict our attention here to such isotropic materials, subjected to laminar shearing displacements. The laminæ which glide over one another will then suffer tangential and normal components of stress whichaccording to the theory ${ }^{1}$ - must vary simultaneously at differing percentage rates, showing, for example, different exponential indices during an exponential build up, or relaxation. In general, when the tan. gential component varies as an odd function of time, the normal one must simultaneously vary according to an even function, so that under vibrational displacements in which the former varies with some frequency around a mean value of zero and according to a sine function, the latter must vary simultaneously with double that frequency around a mean value different from zero, and according to a cosine function.

The experiments were actually made on isotropic materials of the visco-elastic type, such as liquid and solid colloids containing rubber, saponified oils, pastes

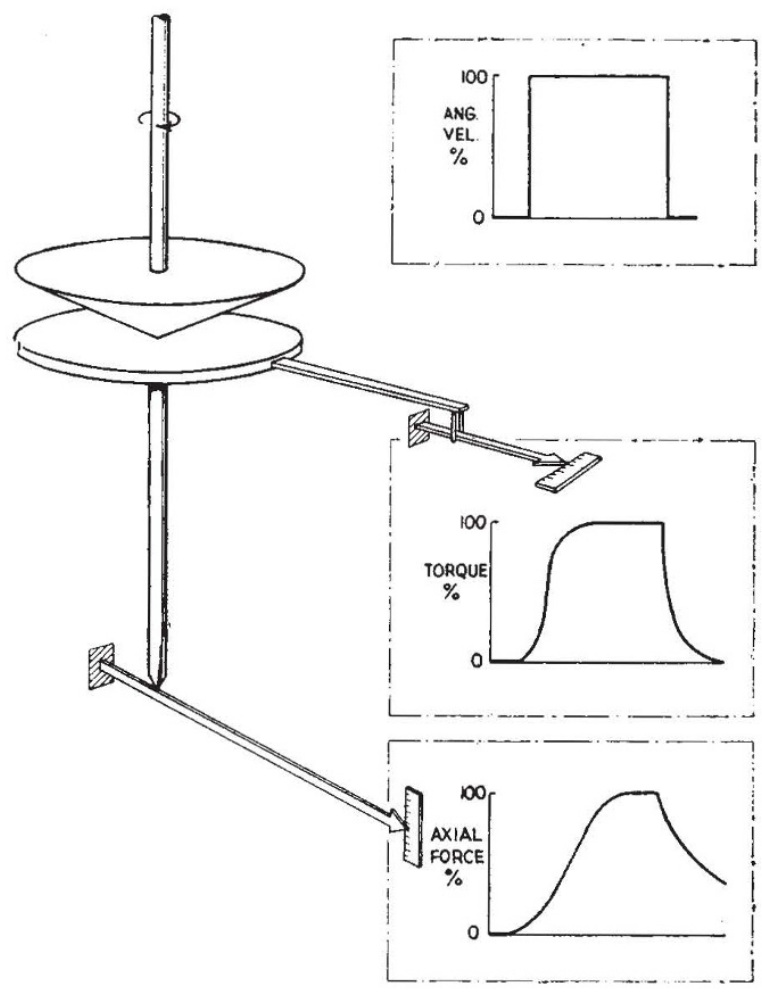

of starch, cellulose acetate, etc., because this type exhibits the anisotropic time effects in an easily accessible range of mechanical actions. (Liquids are understood here as materials which flow under any shearing stress, however small, while solids are capable of maintaining such stresses statically up to a certain limit.) Further, the materials were so selected as to be sensibly incompressible (within the range of applied actions), as this brings about a simplification of the effects here considered.

The accompanying illustration shows on the lefthand side a schematic diagram of the apparatus, and on the right-hand side a typical set of experimental results. The apparatus used was a version of the rheogoniometer ${ }^{2}$ specially designed to impose on a material laminar shearing displacements and to measure along various directions in space the development in time of the stresses. The framework of the apparatus consists of a watchmaker's precision lathe with its axis mounted in a vertical position. Concentric with this axis, and secured respectively in the head-and tail-stock of the lathe, are a circular cone (vertex downwards) and a plane circular disk, which touch one another, and form respectively the roof and the base of a horizontal gap of small height, containing the material under examination. One may impose on the material any laminar shearing displacement desired. For this the cone is rotated about its axis but held rigidly against axial translations, while the disk is kept sensibly at rest by constraints against both rotations and axial translations. The wedge-like shape of the gap then ensures that the material suffers the same amount of shear strain in every differential cell of the gap. The apparatus is equipped with measuring devices. For the strains the angle of rotation of the cone is measured and recorded as a function of time, while for the stresses it is necessary to register the action which the material 
exercises on the disk. To do this, the constraints of the disk are given the form of elastic cantilevers which yield immediately to the action and thereby measure, and record as functions of time, the torque and the axial force, that is, the moment about the axis, and the force perpendicular to the plane of the disk. As the yield of the cantilevers changes the dimensions of the gap, it is necessary to prevent these changes from becoming large enough to disturb the time effects significantly, and to use combinations of mechanical and optical lever systems (not shown in the figure) with a sensitivity of about $10^{-6} \mathrm{~cm}$.

The experimental results are conveniently expressed by three graphs in which are plotted, against time, the angular velocity of the cone and the torque and axial force on the disk, each quantity being expressed in percentage of its maximum value. Assume the material to be incompressible, and the motion one of laminar shear throughout the gap. The torque, $T$, and the axial force, $F$, are then related to the stress components tangential and normal to the plane of the disk by a purely geometrical factor, and as this factor is independent of time, it cancels out when the percentage values are considered as functions of time. Explicitly, introducing cylindrical co-ordinates, we find that in a differential cell at a point $(r, \varphi, z)$ the stress, $P$, has in the plane of the disk the tangential and normal components

$$
P_{\varphi z}=P_{\varphi z}^{\prime} \text { and } P_{z z}=-P_{\varphi \varphi}^{\prime} \log R / r,
$$

whence integrating over the area of the disk we deduce

$$
T=\frac{2}{3} \pi R^{3} P_{\phi z}^{\prime} \text { and } F=\frac{\pi}{2} R^{2} P_{\varphi \Phi}^{\prime},
$$

where the indices attached to $P$ indicate the coordinates involved and the dash denotes the value at a point of the rim of the gap $(r=R)$.

For such an ideal case the three graphs obtained for the macroscopic development in time must be identical with those obtained in every differential cell of the material for the percentages respectively of the strain velocity, and of the stress components tangential and normal to the plane of the disk. (The height of the gap is chosen sufficiently small to make the forces of inertia negligible under the motions of the material here considered.)

In one type of experiment we used visco-elastic liquids; solids are more troublesome for this type of experiments, since in the build-up period they tend to break under the large strains required, and in the relaxation period show inconveniently slow changes of stress. We were concermed with the time effects encountered in the build-up, and in the relaxation of the various components of stress during periods of stationary rates of laminar shearing displacements. It might appear that during these periods any liquid would run out of the gap. How. ever, in the conditions of shear considered, the viscoelastic liquids used here develop hoop-stresses sufficiently strong to form a cylindrical free boundary, and prevent the running out. At first the material was in its ground-state, and the cone and disk were at rest. Next we started a period of build-up of stress by keeping the disk at rest and suddenly imparting to the cone a rotation with an angular velocity that was maintained throughout the period at a constant level sufficiently high to bring about measurable torques and axial forces on the disk. Finally we proceeded to a period of relaxation of stress by suddenly arresting the motion of the cone and keeping the cone and the disk at rest henceforth. Both periods were covered by a photographic record, and a typical set of results is shown on the right-hand side of the figure in the three graphs. The actions applied to the cone are seen in the graph of the angular velocity, and the anisotropic time effects in the differences between the graphs of the torque and axial force. Interpreting the graphs for every differential cell of the material, we find that the strain velocity is exactly synchronized with the action on the cone, while the stress components are delayed, and show anisotropic time effects, the percentage rates of build-up and relaxation being different as between the components tangential and normal to the plane of the disk.

In a second type of experiment we used viscoelastic materials, both liquid and solid, and proceeded as in the first type, except that the shearing displacement imposed by the rotation of the cone was now varied harmonically in time, with a frequency and amplitude chosen sufficiently high to give measurable torques and axial forces. The results obtained so far showed that, in this case, the torque, and the tangential component of stress, varied around a mean value of zero with the same frequency as the imposed displacement, while the axial force and the normal component varied around a mean value different from zero with twice that frequency. The phase and amplitude relations are still being investigated.

The experiments thus show that anisotropic time effects are produced even in isotropic materials during transitions between shear-free and sheared states, and that in the case of laminar shearing displacements the tangential and normal components of stress conform to the theory, showing simultaneously different times of build-up under a stationary rate of displacement, different times of relaxation under constantly maintained displacement, and different frequencies of vibrations under harmonically varying displacements.

The authors' thanks are due to the Director of Research at the Shirley Institute for the opportunity of performing this work and permission to publish it.

${ }^{1}$ Weíssenberg, K., Arch. Sci. Phys. et Nat., 17, 1 (1934); Nature, 159,310 (1947).

${ }^{2}$ Weissenberg, K., Conf. British Rheologists' Club, London, 1946.

\section{DIELECTRIC CONSTANT AND PIEZO-ELECTRIC RESONANCE OF BARIUM TITANATE CRYSTALS \\ BY BERND T. MATTHIAS \\ Laboratory for Insulation Research, Massachusetts Institute of Technology}

$T$ HE growth of barium titanate single crystals and the ferro-electric behaviour of their pseudocubic modification have been describsd earlier ${ }^{1,2}$, and more detailed data can now be given.

There can be obtained at least four modifications of barium titanium compounds, among which the hexagonal and the pseudocubic ones definitely are stoichiometrically $\mathrm{BaTiO}_{3}^{1,2,3}$. The dielectric constant of the hexagorial modification is comparatively low, namely, between 100 and 200. The temperature characteristic shows two small peaks near $0^{\circ} \mathrm{C}$. and $100^{\circ} \mathrm{C}$, at which points maxima of the dielectric constant for the pseudocubic crystals have been found ${ }^{3}$. 